\title{
Magnetic Oil Thermal Behavior under Electromagnetic Induction for Energy Efficient Heating System Design
}

\author{
Ahmet Kucukkomurler ${ }^{1}$, Ramazan Selver ${ }^{2}$ \\ ${ }^{1}$ Suleyman Demirel University Engineering and Architectural Faculty Computer Engineering Department, Isparta, Turkey; \\ ${ }^{2}$ Suleyman Demirel University Engineering and Architectural Faculty Mechanical Engineering Department, Isparta, Turkey. \\ Email: \{akk, rselver\}@mmf.sdu.edu.tr \\ Received February $2^{\text {nd }}, 2010$; revised March $28^{\text {th }}, 2010$; accepted April $3^{\text {rd }}, 2010$.
}

\begin{abstract}
This study describes thermal behavior of magnetic lubricant oil under electromagnetic induction. Experimental set up include oil pump, oil tank, induction heating unit, and heat exchanger. It is a closed loop system where the oil pump circulates oil through oil tank to the system, at the same time induction heating unit heats up to the heat exchanger where the lubricant oil thermal behavior is examined. The unit has been largely studied and tested both magnetic and regular motor oil in a laboratory environment and promising results have been obtained for an actual indoor floor to space heating system design.
\end{abstract}

Keywords: Electromagnetic Induction, Electroheat, Lubricant Oil, Heat Transfer, Fluid Dynamics, Induction Heated Iron, Radiant Heater

\section{Introduction}

Induction heating $(\mathrm{IH})$ applied systems are developed using electromagnetic induction which was first discovered by Michael Faraday in 1831. Electromagnetic induction refers to the phenomenon by which electric current is generated in a closed circuit by the fluctuation of current in another circuit placed next to it. The basic principle of induction heating, which is an applied form of Faraday's discovery, is the fact that AC current flowing through a circuit affects the magnetic movement of a secondary circuit located near it. The fluctuation of current inside the primary circuit provided the answer as to how the mysterious current is generated in the neighboring secondary circuit. Faraday's discovery led to the development of electric motors, generators, transformers, and wireless communications devices. Its application, however, has not been flawless. Heat loss, which occurs during the induction heating process, was a major headache undermining the overall functionality of a system. Researchers sought to minimize heat loss by laminating the magnetic frames placed inside the motor or transformer. Faraday's Law was followed by a series of more advanced discoveries such as Lentz's Law. This law explains the fact that inductive current flow inverse to the direction of changes in induction magnetic movement [1].

Heat loss, occurring in the process of electromagnetic induction, could be turned into productive heat energy in an electric heating system by applying this law. Many industries have benefited from this new breakthrough by implementing induction heating for furnacing, quenching, and welding [2].

In these applications, induction heating has made it easier to set the heating parameters without the need of an additional external power source. This substantially reduces heat loss while maintaining a more convenient working environment. Absence of any physical contact to heating devices precludes unpleasant electrical accidents. High energy density is achieved by generating sufficient heat energy within a relatively short period of time.

The demand for better quality, safe and less energy consuming products is rising. Products using IH include electronic rice cookers, pans and ovens. Safe, efficient and quick heating appliances attract more customers [3]. This study describes using induction heating system to heat lubricant oil up in a close loop floor to space heating system which will be an alternative environmental, low cost, energy efficient heating system for indoor usage. 


\section{Types of Electrical Heating Process}

Prior to describing indoor induction heating system, some types of electric process heating are explained below to help you understand normally used heat sources [4].

The types of electric heating systems are as follows:

1) Resistance Heating;

2) Conduction Heating;

3) Infrared Radiation Heating;

4) Induction Heating;

5) Dielectric Hysteresis Heating;

6) Electric Arc Heating;

7) Plasma Heating;

8) Electron Beam Heating;

9) Laser Heating.

Resistance heating is the most common type of electric process heating. It uses the relationship between the voltage and current of resistance in Joule's Law [5]. Conduction heating exploits the heat energy generated when an object is placed between two electric poles, which is another application of Joule's Law. In this case, however, a different relationship exists between voltage and current, especially when the circuit current is high, because the object itself contains both resistance and inductance features.

The main idea of this study is induction heated iron, which is a combination of electromagnetic induction, the skin effect, and the principle of heat transfer. In short, induction heating refers to the generation of heat energy by the current and eddy current created on the surface of a conductive object (according to Faraday's Law and the skin effect) when it is placed in the magnetic field, formed around a coil, where the AC current flows through (Ampere's Law) [6]. Detailed descriptions of induction heating system are presented in the following sections of the study.

\section{Indoor Electromagnetic Floor to Space Induction Heating System Design}

Indoor induction heating system principle schematic diagram and actual experimental setup picture are shown in Figure 1 and 2 respectively. System has 4 major blocks and the types of blocks are as follows:

1) Oil Tank

2) Oil Pump with DC Servo

3) Induction Heating Unit

4) Heat Exchanger

Close loop system has oil pump which circulates lubricant oil through heat exchanger to the tank. When oil is circulated by the pump, simultaneously induction heating unit is turned on and started to heat up the heat exchanger. While lubricant oil travels through heat exchanger absorbs heat and accumulate heat content on the oil tank. Experimental setup photo can be seen from Figure 2.

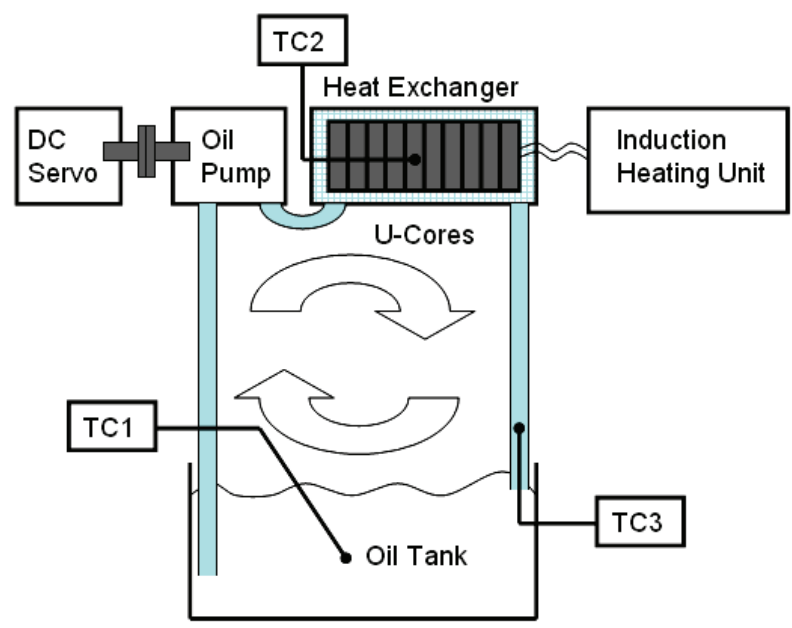

Figure 1. Electromagnetic induction lubricant oil heating system principle schematic

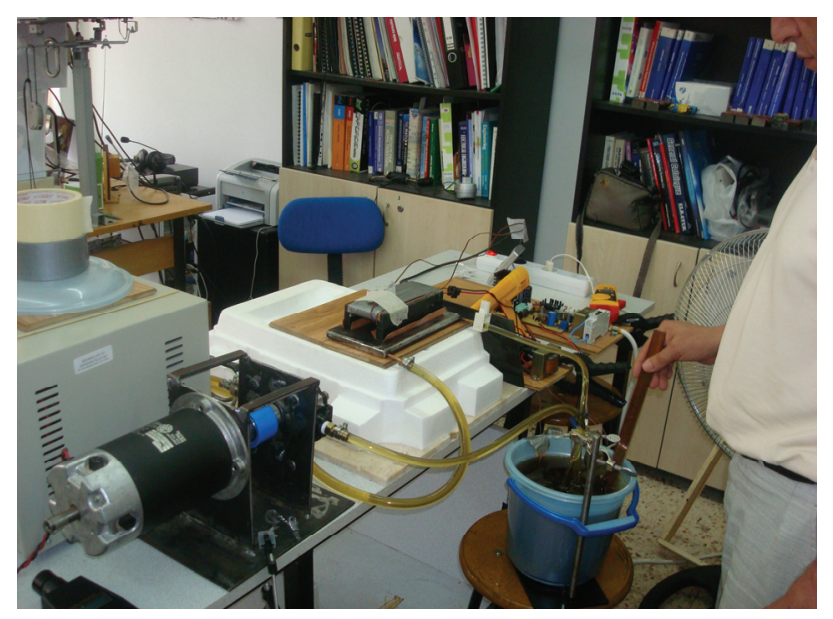

Figure 2. Electromagnetic induction lubricant oil heating system experimental setup

While the experiment is running three different temperature data is recorded. First one is the heat exchanger surface temperature (TC2), second one is lubricant oil temperature in the tank (TC1), and third one is the lubricant oil input temperature to the oil tank (TC3).

Electromagnetic induction unit transformer has been design using ferrite $\mathrm{U}$ cores which has included $12 \mathrm{U}$ cores in a one row and picture with U Cores and Heat Exchanger can be both seen in Figure 3. Primary coil is surrounded on U Cores body on the top and secondary coil is only the heat exchanger by itself. Number of ferrite $U$ cores has been chosen power of the electromagnetic induction unit accordingly.

\section{Indoor Induction Heating Theory, System Performance and Test Results}

Alternate power supply and concept of flux and eddy current circuit is shown in Figure $\mathbf{4}$ at the top and, Ferrite-core, 


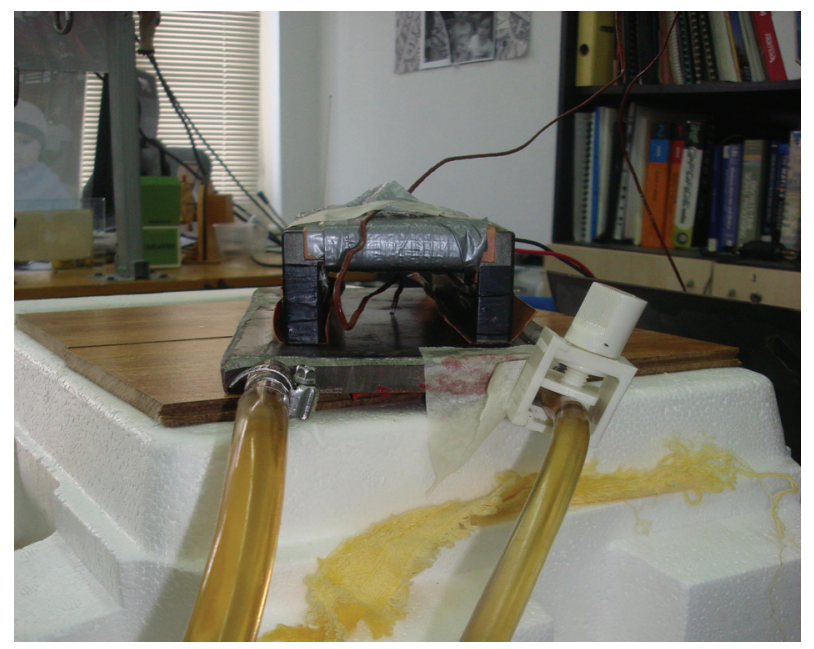

Figure 3. U-Core and heat exchanger from experimental setup

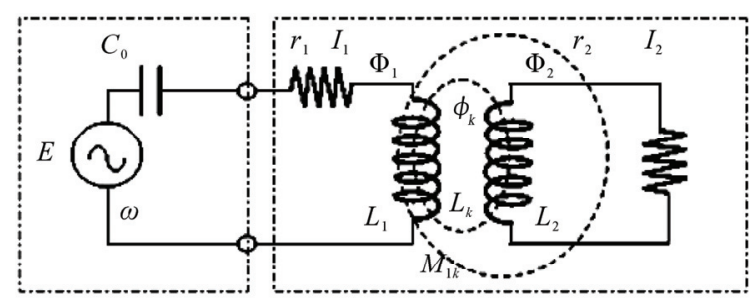

Power Supply $\quad$ Flux and Eddy Current Circuit

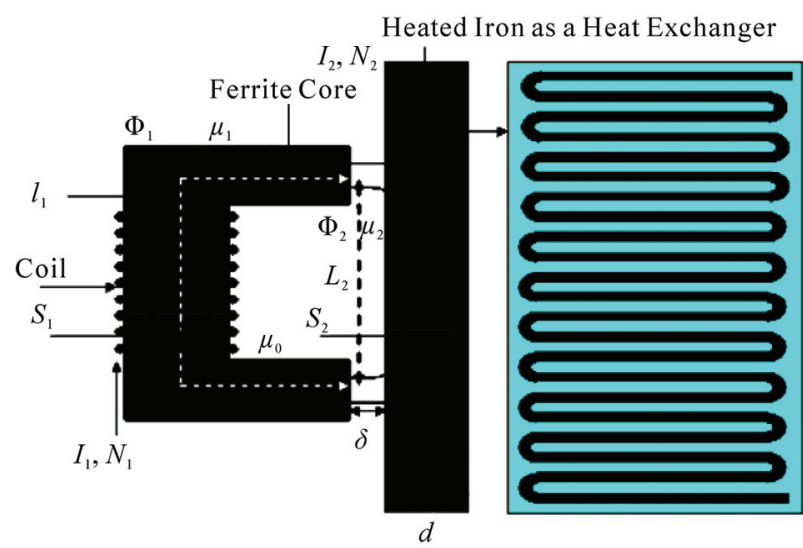

Figure 4. EMI-Heater system principle circuit schematic and induction transformer with heated iron as a heat exchanger

induction heated iron as heat exchanger and magnetic flux is shown in Figure $\mathbf{4}$ at the bottom respectively. The cross-section $S_{1-2}$ of the flux box surrounded and controlled with the parameters thickness $d$, permeability $\mu$, and volume resistivity $\rho$ of the iron [7]. The basic formulas of self $\&$ mutual inductances of eddy current distributions are calculated from the magnetic flux resistances. Changing above four parameters, the phenomena of induction heated iron are investigated using the equation of complex resistance circuits.
Theory behind the induction heated iron has been implemented to heat lubricant oil and promising results have been obtained actual floor to space heater. First magnetic motor oil (Castrol Magnetec 10W-40) has been circulated in the system to see that magnetic property of the oil will observe more heat content then regular motor oil (Castrol 10W-40) which is used as secondary lubricant oil on the experiment to see the difference between them. Both experiments have been performed under room temperature $25{ }^{\circ} \mathrm{C}$ Celsius and oil tank has fill up 3.7854118 liters (a gallon) of lubricant oil in the system on both experiments which have been performed 700 rpm for both liquids to keep the same amount of liquid travel inside the heat exchanger at the same time period. Each experiment has been run 90 minutes and temperature data collected respectively. Results are depicted in Figure 5 (excluded very first data, temperature jumps very quickly because the nature of induction heating) which are the temperature measurement by thermocouples (from Figure 1) TC1-oil tank, TC2-surface of the heat exchanger (in the middle), and TC3-oil tank input.

Experiments are clearly shows that magnetic motor oil much better thermal observant then regular motor oil. Results are clear that magnetic property of the lubricant makes better heat observer, however the amount of the magnetization is questionable in terms of clogging the system. This has to be further investigated to find out optimum level of magnetic property for the lubricant oil. Power consumption is the induction heating unit by itself is only 330 Watts which makes attractive heating system when compare around the electrical heaters. The system can easily turn to be a floor to space heater. This can be done by replacing oil tank to the radiant tubes which caries the lubricant oil under the floor. Isolated room (under $10 \mathrm{~m}^{2}$ ) can be heated only 330 Watts power consumption

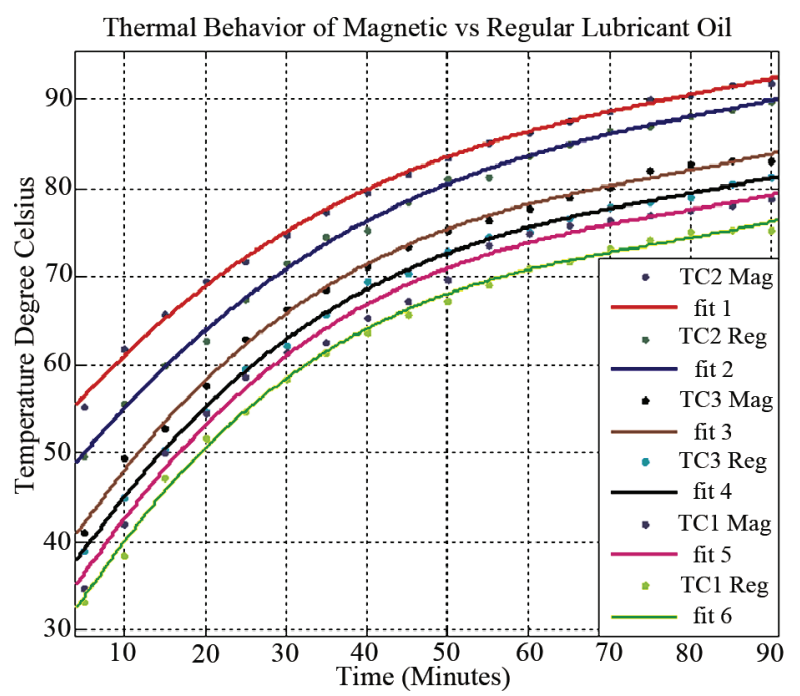

Figure 5. Temperature data for both magnetic vs regular lubricant oil 
from the oil tank temperature data calculations. This makes the heating system energy efficient and comparable to all other electrical heaters available on the market.

\section{Acknowledgements}

The authors would like to thank Valentine PETRAN for his support and valuable discussions.

\section{REFERENCES}

[1] "Heat Treating," Metals Handbook, 9th Edition, American Society for Metals, Vol. 4, 1981.

[2] S. L. Semiatin and D. E. Stutz, "Induction Heat Treating of Steel," American Society for Metals, 1986.
[3] A. Kucukkomurler, "Environmental, Low Cost, Energy Efficient Electromagnetic Indoor Induction Space Heating System Design," IEEE Eurocon 2009, Saint Petersburg, 2009, pp. 1604-1606.

[4] C. A. Tudbury, "Basics of Induction Heating," John F. Rider, Inc., New Rochelle, New York, 1960.

[5] "Heat Treating," ASM Handbook, ASM International, Vol. 4, 1991.

[6] P. Sergeant, et al., "Thermal Analysis Magnetic Shields for Induction Heating," IET Electric Power Applications, Vol. 3, No. 6, 2009, pp. 543-550.

[7] J. Acero, et al., "Domestic Induction Appliances," IEEE Industry Applications Magazine, Vol. 16, No. 2, 2010, pp. 39-47. 